\title{
Incorporando al Otro: un examen histórico de los procesos productivos del pueblo Yánesha de la Selva Central
}

\author{
Alex Avendaño \\ Bachiller en Antropología de la Pontificia Universidad Católica del Perú \\ Miembro del Grupo de Antropología Amazónica (GAA-PUCP)
}

\section{Resumen}

Un tema común entre las investigaciones sobre las economías indígenas amazónicas ha consistido en el cambio de su carácter 'tradicional' por procesos de inserción al mercado. El caso del pueblo Yánesha, que habita el valle del Palcazú, no ha sido la excepción. En este espacio, al desarrollo de la producción ganadera comercial y el establecimiento de tres grandes áreas naturales protegidas, le han seguido proyectos en torno a la sostenibilidad ambiental en los últimos 30 años. En esa línea, el presente ensayo pretende tomar una ruta distinta para la interpretación sobre el cambio económico y social de este pueblo. Basándose en la revisión de tres momentos históricos y en una salida de campo exploratoria, este texto atiende los cambios en los procesos productivos, y contempla modos de relacionamiento con la alteridad de las poblaciones indígenas amazónicas, caracterizados por una disposición de 'apertura al otro'. De esta manera, se argumentará la necesidad de cuestionar las nociones de inserción al mercado como una simple integración pasiva; $y$, asimismo, de aproximarnos teóricamente a las maneras en que los miembros de las poblaciones Yánesha incorporan los elementos foráneos a su vida social mediante la práctica.

\section{Palabras clave}

Procesos productivos, alteridad, Yánesha, inserción al mercado, cosmologías indígenas. 


\title{
Incorporating the Other: a historical examination of the productive processes of the Selva Central's Yánesha people
}

\author{
Alex Avendaño \\ Bachelor in Anthropology from the Pontifical Catholic University of Peru \\ Member of the Amazon Anthropology Group (GAA-PUCP)
}

\begin{abstract}
A common theme among research on Amazonian indigenous economies has been the change of its 'traditional' character by processes of market insertion. The case of the Yánesha people, that inhabits the Palcazu valley, has not been the exception. In this space, the development of commercial livestock production and the establishment of three large protected natural areas have been followed by projects around environmental sustainability in the last 30 years. The present essay intends to take a different route for the interpretation on the economic and social change of this town. Based on the review of three historical moments, and an exploratory field trip, this text deals with the changes in the productive processes, contemplating ways of relating to the otherness of the indigenous Amazonian populations, characterized by a disposition of 'openness to the other'. In this way, the need to question the notions of insertion into the market as a simple passive integration will be argued; and, also, to approach theoretically to the ways in which the members of the Yánesha populations incorporate the foreign elements to their social life through practice.
\end{abstract}

\section{Keywords}

Productive processes, alterity, Yánesha, market insertion, indigenous cosmologies. 


\section{Introducción}

La relación que los pueblos indígenas de la Amazonía peruana han establecido con la miríada de ecosistemas que habitan, mediante sus procesos productivos, ha estado en el ojo de numerosas investigaciones desde la antropología. Los primeros esfuerzos en la producción etnográfica estuvieron orientados a la descripción y el análisis de los 'sistemas de subsistencia' y estrategias productivas indígenas (Calderón Pacheco, 2012, p. 238). En los últimos veinte años, la preocupación ha girado hacia los procesos históricos y contemporáneos de inserción a la economía de mercado (Barclay, 1991; Calderón Pacheco, 2012; Smith \& Wray, 1996). ¿Hacia dónde se dirigen los pueblos indígenas con el conjunto de cambios que entraña esta vinculación? Las interpretaciones han variado desde la desestructuración del sistema de subsistencia hacia el establecimiento de una 'economía mixta de producción para el mercado y el autoconsumo'. Reconociendo la importancia del análisis de la agricultura itinerante, la caza, la pesca y el forrajeo, sostengo que la investigación etnográfica también debe indagar por la posición propia de los sujetos indígenas, en el contexto de su vida social (junto a otras dimensiones centrales como el parentesco o lo político), en torno a la incorporación de objetos, ideas y técnicas foráneas en aquellas actividades productivas. Esto implica alejarse de una concepción pasiva del proceso de inserción al mercado para poder enfocarse en las prácticas con que las poblaciones indígenas han apropiado los elementos del mismo.

A través de su historia, el pueblo Yánesha de la Selva Central peruana ha atravesado momentos donde sus relaciones económicas con la población colonizadora y el Estado tomaron diferentes formas. A partir de la década de 1980 y de la iniciativa pionera del Proyecto Especial Pichis Palcazú (PEPP), el valle del Palcazú y sus habitantes Yánesha se han encontrado con las intervenciones de proyectos estatales y privados bajo modelos de sostenibilidad ambiental (Benavides \& Pariona, 2002; GarcíaCalderón, Huerto, Quispe, \& Tamariz, 2011; Larsen, 2016). Si retrocediésemos hasta los inicios del siglo XX, podría afirmarse que los intercambios desiguales de la fuerza de trabajo indígena en las haciendas ganaderas o cafetaleras de la región marcó el quiebre paulatino entre una economía 'tradicional' y otra 'integrada' (Santos Granero \& Barclay, 1995). Sin embargo, encuadrar el proceso en estos términos implica enceguecerse ante la relación activa que los miembros de las familias Yánesha han establecido con especies, artefactos y técnicas originalmente foráneas desde una época tan temprana como el de la colonización española de los valles intermontanos del piedemonte andino. 
¿Por qué no intentar seguir el proceso histórico de inserción a la economía de mercado desde la propia posición que la gente Yánesha puso en la marcha? Este ensayo se enfocará en sus procesos productivos, que se han encontrado en el epicentro de las relaciones con misioneros, colonos y el Estado peruano. De esta manera, se revisarán tres momentos específicos en tres siglos de su historia en la Selva Central, basándose en fuentes bibliográficas y una salida exploratoria de campo a la C.N. Siete de junio del valle del Palcazú realizada a fines de agosto del 2018'; y partirá desde los modos de relacionamiento con la alteridad inscritos en las cosmologías de los pueblos indígenas de la cuenca amazónica (Viveiros De Castro, 2004).

\section{La economía más allá de lo convencional: relaciones con la alteridad y praxis productiva}

En un artículo que revisita a Lévi-Strauss, Jean-Pierre Chaumeil (2010) recapituló, a través del 'mito de los mellizos', las ideas acerca de un modelo de la relación de los pueblos indígenas amazónicos con la alteridad, en el marco de sus cosmologías. El autor constata que, en la producción etnográfica, este se caracteriza por consistir en una disposición para la incorporación activa de aquellos elementos pertenecientes a este otro, expresándose, principalmente, en las transformaciones del cuerpo -por ejemplo, la adopción de la 'ropa de los blancos' en el caso de los Yine (Chaumeil, 2010; Gow, 2007)-; pero también es posible observar esta disposición en sus prácticas económicas a partir de investigaciones etnográficas realizadas entre poblaciones indígenas de distintos grupos en la cuenca amazónica (Arisi, 2012; Brightman, 2012; Walker, 2012; Zanotti, 2016). Estos trabajos comparten la constatación de que los sujetos indígenas buscan asir los objetos foráneos, reapropiándolos en su vida social bajo sus propios términos conceptuales, evitando ser rebasados por las relaciones dentro de los cuales circulan -como el intercambio directo de las mercancías dentro del sistema capitalista (Walker, 2012, p. 142)-. No obstante, antes de continuar, se requiere redefinir 'la economía' del pueblo Yánesha de una manera que exceda los constreñimientos que puede otorgarle una lectura convencional del marco teórico con el que se ha valido la antropología económica.

Al trabajar con una definición sustantiva de la economía, Wilk y Cliggett (2007) optan por evitar circunscribirla al circuito clásico de producción, distribución y consumo, debido al cariz etnocéntrico que pueden adoptar estas categorías. De esta manera, la economía consistiría en "las relaciones entre los seres humanos y el mundo de objetos, ideas e imágenes producidas por humanos" (Wilk \& Cliggett, 2007, p. 36). Incluso, si no abandonamos las categorías ya mencionadas para los propósitos de análisis en la investigación etnográfica, resulta importante otorgarle centralidad a la definición amplia de estos autores: el eje crucial en las actividades y prácticas que comprenden las fases clásicas es la vinculación de los seres humanos mediante los artefactos que producen. Por otro lado, los autores excluyen, deliberadamente, al entorno biofísico de esta definición, posición que ha sido refutada por Hirsch. En su respuesta, este último autor apuntó la intervención activa de las poblaciones indígenas amazónicas para transformar los diversos ecosistemas de la región desde hace siglos $(2005$, p. 373).

1 Esta salida exploratoria tuvo una duración de cinco días, se basó en un diseño no estructurado y estuvo enfocado en el tema general de la economía de las familias Yánesha del sector 'Pampacocha' de la comunidad. Asimismo, se enmarca en el Programa de Apoyo a la Iniciación en la Investigación (PAIN) de la Pontificia Universidad Católica del Perú (PUCP). 
Asimismo, un cuerpo de investigaciones ha mostrado que no puede deslindarse el estudio de la economía indígena en la Amazonía sin tomar en cuenta las relaciones activas con el entorno y sus elementos no-humanos, que cobra una importancia más allá de los aspectos materiales de la producción (Descola, 1988; Santos Granero, 2004; Smith \& Wray, 1996; Viveiros De Castro, 2004). En este campo de encuentros entre los humanos y los no-humanos, se vuelve imperativo investigar los procesos productivos desde un enfoque etnográfico.

En la aproximación etnográfica a los procesos productivos del pueblo Yánesha, la noción de 'práctica' cobra bastante importancia. Como señala Sherry Ortner (1984), este término ha tenido una preponderancia creciente en la teoría antropológica de la década de 1980 -en respuesta a intentos de subyugar toda la complejidad de la vida social a definiciones totalizantes como la cultura. En este sentido, Wilk y Cliggett han apuntado cómo las presunciones sobre la naturaleza fundamental que rige el comportamiento económico de los seres humanos varían, idealmente, desde el completo individualismo hasta el sometimiento de la persona al grupo social (o la cultura) según las distintas vertientes de la teoría social moderna (Wilk \& Cliggett, 2007, p. 42). Habiendo escrito esto, ¿cómo resolver la tensión entre las prácticas productivas -y económicas en general- de los sujetos Yánesha y aquel carácter totalizante que puede implicar la noción de 'cosmología' u 'ontología'? La respuesta que sustento consiste en entender las cosmologías indígenas amazónicas (y la de los Yánesha) como un habitus. Es decir, conceptualizarlo como un sistema de disposiciones duraderas que orientan la práctica, mas no la determinan (Bourdieu, 2008). Al teorizar sobre las relaciones entre los humanos y el entorno en sus procesos productivos, autores como Maurice Godelier y Philippe Descola han sostenido cómo no puede separarse -más allá de propósitos analíticos- lo 'ideal' de lo 'material' en las prácticas productivas de los sujetos (Descola, 1988; Godelier, 1990). Bajo este planteamiento, se entiende que, a través de estas interacciones entre los humanos y no-humanos, que pueblan un cosmos común, se vuelven inteligibles las disposiciones de los primeros respecto a estas relaciones.

En la aproximación etnográfica a los procesos productivos del pueblo Yánesha, la noción de 'práctica' cobra bastante importancia. Como señala Sherry Ortner (1984), este término ha tenido una preponderancia creciente en la teoría antropológica de la década de 1980 -en respuesta a intentos de subyugar toda la complejidad de la vida social a definiciones totalizantes como la cultura. En este sentido, Wilk y Cliggett han apuntado cómo las presunciones sobre la naturaleza fundamental que rige el comportamiento económico de los seres humanos varían, idealmente, desde el completo individualismo hasta el sometimiento de la persona al grupo social (o la cultura) según las distintas vertientes de la teoría social moderna (Wilk \& Cliggett, 2007, p. 42). Habiendo escrito esto, ¿cómo resolver la tensión entre las prácticas productivas -y económicas en general- de los sujetos Yánesha y aquel carácter totalizante que puede implicar la noción de 'cosmología' u 'ontología'? La respuesta que sustento consiste en entender las cosmologías indígenas amazónicas (y la de los Yánesha) como un habitus. Es decir, conceptualizarlo como un sistema de disposiciones duraderas que orientan la práctica, mas no la determinan (Bourdieu, 2008). 
Al teorizar sobre las relaciones entre los humanos y el entorno en sus procesos productivos, autores como Maurice Godelier y Philippe Descola han sostenido cómo no puede separarse -más allá de propósitos analíticos- lo 'ideal' de lo 'material' en las prácticas productivas de los sujetos (Descola, 1988; Godelier, 1990). Bajo este planteamiento, se entiende que, a través de estas interacciones entre los humanos y no-humanos, que pueblan un cosmos común, se vuelven inteligibles las disposiciones de los primeros respecto a estas relaciones.

\section{El proyecto misionero y el hierro en la selva [1709 - 1742]}

Durante el siglo XVII, la orden franciscana intentó reducir en numerosas ocasiones a las poblaciones indígenas de la Selva Central sin éxito alguno. Esta tendencia cambió cuando Francisco de San Joseph asumió la dirección de la orden franciscana, fundando siete misiones y tres doctrinas donde se concentró, a partir de 1709, a la población Yánesha y asháninka de distintos lugares pertenecientes a las conversiones de Huánuco, Tarma y Jauja (Santos Granero, 2004, p. 191). Durante un lapso de tres décadas, las misiones generaron impactos drásticos en distintos aspectos de la vida social de los Yánesha que se incorporaron a dichas misiones. Así, el conjunto de la población, que en ese entonces habitaban en los valles intermontanos de los actuales distritos de Oxapampa, San Luis de Shuaro, Perené y Chanchamayo, atravesó un conjunto de cambios a nivel socio-espacial, socioeconómico y demográfico -siendo este último el más importante debido a los estragos causados por las epidemias entre la población indígena (Santos Granero, 2004, p. 192). En este periodo, la economía Yánesha cambió en un sentido doble. A largo plazo, debido a la adquisición de nuevas especies, objetos y tecnologías foráneas; y temporalmente, a raíz del ordenamiento del tiempo mediante el trabajo en las misiones.

Las misiones contribuyeron a una serie de cambios duraderos en la economía Yánesha. En primer lugar, estas proveyeron utensilios de metal, muy demandados entre la población indígena; e introdujeron las tecnologías para la forja de herramientas entre los Yánesha (Santos Granero, 2004, pp. 197-198). La importancia del aprendizaje de la forja de herramientas de hierro radicó en la incorporación de las herrerías al complejo político-religioso de templos, asociados a la figura del kornesha, que formaron parte del sistema sociopolítico Yánesha y se mantuvieron hasta comienzos del XX (Santos Granero, 1994, 2004). Otros elementos nuevos introducidos por las misiones fueron especies para el cultivo (plátano, cítricos y caña de azúcar) y la crianza (ganado vacuno y pecuario) que continuaron dentro de los procesos productivos de las familias Yánesha tras un siglo de la clausura de la región a los colonizadores (Santos Granero, 2004, p. 202). Como puede observarse, mediante este conjunto de adquisiciones, los Yánesha demostraron su disposición de apertura para incorporar activamente las especies, artefactos y técnicas foráneas a los procesos productivos preexistentes. Con el caso de los templos-herrerías, esta excedió el entendimiento convencional de 'la producción' como una actividad acotada de transformación de la materia para involucrarse con el papel que los kornesha pasaron a jugar en la cosmología del pueblo Yánesha. Sin embargo, en las haciendas se revelaría otra cara, más tensa, de este proceso de incorporación de elementos foráneos. 
$\mathrm{Al}$ interior de las misiones, los miembros de las familias indígenas fueron asignados con un conjunto de actividades adicionales (trabajo en las tierras o talleres de la misión) destinadas a suplir las necesidades de los misioneros -y de la misión en general- y la producción comercial (coca, caña de azúcar y aguardientes, y algodón y textiles), encauzando el tiempo del que disponían y distribuyéndolo de acuerdo a los intereses misionales (Santos Granero, 2004, p. 198). En un contexto más contemporáneo, Marc Brightman reflexionó acerca de cómo el intento de los misioneros para imponer su autoridad, mediante la estructuración del tiempo en semanas y años, fue cooptado por la ideología del ascetismo que guiaba a los Trio del sur de Surinam (2012, p. 564). Relacionándolo a lo escrito sobre diferentes momentos en la historia de las relaciones económicas de los Yánesha con la población colonizadora, puede afirmarse que la valoración de la autonomía entre los primeros habría entrado en conflicto con el intento de regimentar el tiempo -y la imposición de la autoridad que implicaba- de los segundos (Benavides \& Pariona, 2002; Santos Granero, 2004; Smith \& Wray, 1996). El descontento por este intento de control en las haciendas misioneras y la disminución poblacional por las epidemias terminó explotando en la rebelión multiétnica de Juan Santos Atahualpa en 1742; y acabó con el repliegue de los colonos y del Estado hasta mediados del siglo XIX.

\section{La ganadería Yánesha en las comunidades nativas [1950 - 1970's]}

Al comienzo del proceso de recolonización de la Selva Central, iniciado en 1847 con el establecimiento del Fuerte San Ramón, le siguieron sucesivos momentos de reconstitución de las misiones y despojo en el territorio Yánesha del piedemonte (Santos Granero, 2004; Santos Granero \& Barclay, 1995). En consecuencia, el grueso de las familias Yánesha migraron a la zona de Villa Rica y al valle del Palcazu (ubicado al norte de la primera). No obstante, no fueron el único grupo que se estableció en el valle a inicios del siglo XX. Una serie de familias de colonos europeos, provenientes de la colonia de Pozuzo, se asentaron en estos espacios y establecieron haciendas ganaderas (Santos Granero \& Barclay, 1995; Smith, 1983). De forma general, la relación económica entre las familias Yánesha y de hacendados consistió en el intercambio desigual de la fuerza de trabajo de los miembros de las primeras mediante el sistema de habilitación y enganche. Por un lado, los Yánesha se insertaron como peones en la producción de café comercial en las haciendas cafetaleras de Villa Rica durante los periodos de cosecha (Santos Granero \& Barclay, 1995, p. 276). Mientras tanto, los hacendados ganaderos del Palcazú se aseguraron la fuerza de trabajo de los Yánesha, mediante el sistema de 'contratas', valiéndose de la deuda y consiguiendo la apertura de pastizales entre las décadas de 1950 y 1970 (Santos Granero \& Barclay, 1995, pp. 280-281).

No obstante, la participación de los Yánesha en los procesos productivos de las haciendas conllevó a su adquisición de las especies comerciales de ambas zonas. Debido a que la 'parte baja' no es adecuada para el cultivo del café, la principal opción de las familias Yánesha en el valle del Palcazú consistió en la adquisición de ganado vacuno mediante el sistema de crianza de ganado 'al partir' de manera creciente desde la década de 1960 -pudiendo constituir hatos propios (Santos Granero \& Barclay, 1995; Smith, 1983). 
Frente a los costos y la fuerza de trabajo que demanda la crianza de ganado, los Yánesha recurrieron a algunas alternativas de su organización productiva -las asociaciones interfamiliares informales y la ganadería comunal (que solo pudo consolidarse en una comunidad) (Santos Granero \& Barclay, 1995, p. 302). Así, una proporción significativa de las familias de comuneros Yánesha en las comunidades del Palcazú dedicaron mayor tiempo y esfuerzos a la ganadería comercial. No obstante, también se han planteado las desventajas que puede generar, tales como la disminución de la producción para el autoconsumo, el aumento en la dependencia del mercado y el deterioro del conjunto de procesos productivos por presiones ecológicas y ambientales (Santos Granero \& Barclay, 1995, pp. 304-305).

Tras la constitución del Congreso Amuesha y las comunidades nativas del valle del Palcazú durante el Gobierno de las Fuerzas Armadas, esta primera organización intentó fortalecer la ganadería Yánesha en un contexto de pugnas con las familias de hacendados por la autonomía en la comercialización del ganado. Este es el caso del Comité Agroyánesha, que incorporó, tempranamente, a un cuerpo de técnicos extranjeros con conocimientos en torno a la crianza del ganado vacuno (Santos Granero \& Barclay, 1995; Smith, 1983). A pesar de que, en la década posterior, el $\mathrm{PEPP}^{2}$ planificó una producción sostenible de los pastizales, este también enfatizó un modelo de desarrollo sostenible que esperaba desplazar la atención de las familias Yánesha hacia las actividades forestales (Staver, Simeone, \& Stocks, 1994, p. 155). De esta manera, el Estado volvió a entrar en las relaciones entre los Yánesha y el mercado, mediante un proyecto regido bajo el paradigma naciente del desarrollo sostenible y la sostenibilidad ambiental.

\section{Carretera, madera y proyectos forestales [1985 - 2010's]}

Con la formulación y ejecución de los distintos componentes del PEPP, una serie de procesos simultáneos convergieron en el Palcazú (a mediados de la década de 1980): la culminación de la carretera Villa Rica-Iscozacín en 1985, la creación de tres grandes áreas naturales protegidas de diferente denominación y el primer proyecto con fines de desarrollo sostenible en el valle -la experiencia pionera de la Cooperativa Forestal Yánesha (COFYAL) (Benavides \& Pariona, 2002; Hartshorn \& Stocks, 1993; Staver et al., 1994). En este momento del proceso de inserción de los Yánesha al mercado, tres hechos resaltaron. Primero, el paradigma de la sostenibilidad atravesó este momento de inserción de los Yánesha al mercado. En los procesos productivos, esto debió suponer el comienzo del aprendizaje de un cuerpo de herramientas, técnicas y tecnologías para el 'desarrollo sostenible'. En segundo lugar, la consecución de la carretera disminuyó la capacidad de los 'patrones ganaderos' para monopolizar la comercialización del ganado. Por último, esta vía de transporte que atravesaba las comunidades Yánesha permitió tanto la entrada de los comerciantes madereros de Oxapampa para la extracción forestal como la posibilidad de que algunas familias Yánesha establezcan pequeños negocios a lo largo del camino, que atravesaba a sus comunidades (Benavides \& Pariona, 2002).

2 Proyecto Especial Pichis-Palcazú 
La peculiaridad en los procesos productivos alrededor de las actividades de extracción de madera radica en el control por parte del Estado y la comunidad nativa (de ahora en adelante, C.N.) para su realización por parte de las unidades domésticas Yánesha individuales (Benavides \& Pariona, 2002, p. 317). Aunque la COFYAL supuso una iniciativa de organizar alternativamente la extracción y comercialización de la madera, esta languideció entrando en la década de 1990. Algunos autores señalan como causas de fondo el contexto de intensificación de la violencia política, el breve surgimiento de grupos narcotraficantes en el valle y las tensiones entre las lógicas económicas de la cooperativa y de las familias Yánesha (Benavides \& Pariona, 2002; Larsen, 2016).

A partir de la mitad de la década de 1990, hay un esfuerzo del Estado por la reforestación en el valle del Palcazú; y, a partir de la década del 2000, vuelve una serie de proyectos -de menor alcance que el PEPP- bajo el mismo paradigma de la sostenibilidad ambiental (Benavides \& Pariona, 2002; Larsen, 2016). En palabras de Larsen, estaríamos ante el cambio narrativo de la frontera hacia otro de posfrontera -donde se asume el apogeo de las modalidades de gobernanza caracterizadas por el reconocimiento, protección ambiental y salvaguardas sociales (Larsen, 2016, p. 24). El autor muestra cómo, en el valle del Palcazú, la existencia de este régimen de posfrontera oficial no elimina el contexto histórico de las relaciones de poder arraigadas entre las poblaciones Yánesha con los colonos. Este es el caso explorado con los comerciantes madereros, quienes lograron una gobernanza sobre el bosque y sus recursos forestales a partir de intercambios desiguales marcados por la deuda con las comunidades nativas Yánesha (Larsen, 2016, pp. 165-167). Mientras se reconoce la sujeción pasiva y el aspecto asimétrico de esta relación de intercambio, también debe considerársele como el marco, desde el que es posible la construcción de una agencia por parte de los sujetos Yánesha, como Walker argumenta respecto a la situación de los Urarina en el sistema de habilitación-enganche (2012).

En este último decenio, el Estado y sus proyectos para la sostenibilidad ambiental y el desarrollo sostenible marcan muchos puntos clave en la institucionalidad ambiental en el Perú. Por un lado, se tiene la creación del Ministerio del Ambiente (MINAM) y el Servicio Nacional de Áreas Naturales Protegidas del Perú (SERNANP) en el año 2008, y el Servicio Forestal Nacional (SERFOR) en el 2014. Por el otro, también ocurrió el nombramiento de la provincia de Oxapampa por la UNESCO como la Reserva de Biósfera Oxapampa Asháninka Yánesha ${ }^{3}$. Durante una salida exploratoria a la C.N. Siete de junio, en el valle del Palcazú, observé cómo los esfuerzos para gestionar estos espacios de conservación se plasmaban en carteles y construcciones a lo largo de la carretera y en el poblado de Iscozacín; pero también se realizaban en la vida social contemporánea en las comunidades Yánesha.

La Reserva Comunal Yánesha (RCY) es un área natural protegida creada en 1988, con la finalidad de proveer recursos alimenticios - a partir de la caza, por ejemplo- para la población de 10 comunidades Yánesha del valle. Estas se encuentran asociadas en la Asociación para el Manejo de la Reserva Comunal Yánesha (AMARCY), que cuenta con un Plan Maestro para la planificación del uso y manejo de este espacio durante cinco años (SERNANP, 2012).

3 Debido a los esfuerzos en conservación que representaban sus áreas naturales protegidas (Larsen, 2016, p. 57) 
Sin embargo, los esfuerzos estatales para la sostenibilidad ambiental son socavados por el establecimiento de poblaciones de colonos dentro de estos espacios-situación que me fue comentada por mis interlocutores Yánesha en Pampacocha mientras observábamos las colinas y montañas que se dirigen hacia la cordillera de Yanachaga. Por otro lado, cabe resaltar la interacción de los comuneros con la lengua y los procedimientos propios de los proyectos para la sostenibilidad al involucrarse con la vigilancia comunitaria voluntaria del Parque Nacional. Este es el caso de Emilio ${ }^{4}$, un comunero de Pampacocha que me habló sobre los diez días del año que dedicó a esta labor.

La otra cara de la incorporación activa de objetos, ideas y técnicas foráneos se refiere al campo de los procesos productivos agrícolas intervenidos por los proyectos de desarrollo sostenible estatal. El SERNANP ha promovido en las comunidades Yánesha un modelo de producción comercial basado en un cultivo destinado al mercado nacional y mundial: el cacao. Un estudio previo constata que en el sector Pampacocha, el cultivo del cacao promovido por esta entidad se ha llevado a cabo por varios años (García-Calderón et al., 2011). Durante mi visita a la comunidad, una serie de comuneros me comentaban sobre su producción actual de cacao. En otras ocasiones, miraba los granos en los tendales para el secado al transitar en el sector de Villa América. Al acompañar a una familia a su casa, ubicada en medio de las chacras de los terrenos bajos del sector Pampacocha, la primera extensión de cultivos por el que caminamos estaba exclusivamente dedicada a este fruto. Las respuestas que recibí al preguntar sobre estas plantaciones coincidieron en que se trataban de proyectos que llevaban años y habían resultado en la entrega y capacitación en el cultivo de una cuadra de cacao por comunero. Simultáneamente, resulta interesante la disminución en la importancia atribuida a las actividades de crianza de ganado entre los comuneros de Pampacocha -aunque sigue habiendo ganado dentro de la economía familiar. En conclusión, la adquisición de algunas actividades productivas y el declive de otras, en este contexto histórico, abren interrogantes que no pueden ser respondidas sin recurrir a una investigación etnográfica más prolongada.

\section{Conclusiones}

La duda inicial del presente texto giró en torno a la pertinencia de asumir acríticamente la discontinuidad entre una 'economía indígena tradicional basada en la subsistencia' y una 'economía mixta' que combina actividades orientadas al autoconsumo y al mercado. La posición de este ensayo ha sido tomar la distinción como analíticamente correcta; pero, también, de poner en duda la validez de este cambio como una ruptura súbita para los miembros del pueblo Yánesha. Este punto se argumentó señalando su aprehensión de los elementos foráneos a sus procesos productivos mediante el marco de referencia de las disposiciones cosmológicas de los pueblos indígenas de la Amazonía. En los tres momentos históricos, se constatan relaciones de intercambio desigual-que también son relaciones de poder-entre los sujetos Yánesha y los 'otros', conectados a la economía regional. Sean los misioneros, las familias de hacendados o los comerciantes madereros, la sujeción que intentan ejercer, mediante el 'sistema de habilitación enganche', es subvertida, paulatinamente, por la adquisición del conocimiento práctico sobre las especies, artefactos y técnicas y su inserción a los procesos productivos por parte de los Yánesha.

4 El nombre original de mi interlocutor fue cambiado bajo los términos que supone la confidencialidad de los informantes. 
Por otro lado, la inevitable conjunción temática de lo económico y la ecología supone un desafío adicional al ordenamiento de un incipiente campo de estudios sobre economías indígenas amazónicas desde la antropología. Intersectando las preguntas habituales sobre conocer el papel que juegan las poblaciones indígenas en la transformación de sus entornos biofísicos (por ejemplo, la deforestación o conservación de los bosques), la antropología actual tiene la capacidad de introducir dos perspectivas útiles. La primera, desarrollada por la antropología amazónica (a una escala regional), consistiría en un giro a las prácticas y discursos de la gente indígena sobre estas economías, insertando sus modos de socialidad en el análisis y la interpretación de la data etnográfica. En segundo lugar, resulta necesario responder en la investigación etnográfica ante las intervenciones contemporáneas de los diferentes programas estatales y proyectos para la conservación del ambiente y desarrollo sostenible que predominan las economías locales. El motivo recae en los efectos duraderos que el cuerpo de gente organizada tras estas iniciativas (por ejemplo, entidades estatales ambientales u $\mathrm{ONG}$ ) puede tener en la vida social y económica de la población indígena residente en su área de influencia (como a nivel distrital o provincial). Asimismo, también amerita tomar en cuenta la relación de estos nuevos sujetos y entidades económico-políticos con los antiguos. Esto puede ser de vital importancia para entender también los conflictos vigentes entre el Estado peruano y las 'economías ilícitas' (como cadenas de producción de cocaína para narcotráfico) al tomar en cuenta los riesgos para la integridad de los investigadores y de los sujetos de investigación durante el recojo de información.

Finalmente, bajo el establecimiento del paradigma de sostenibilidad ambiental, protección social y 'cierre de las fronteras', el panorama del Palcazú abre muchas preguntas para el transcurso de esta década. De manera específica, las intervenciones del Estado, a partir de los proyectos y medidas gestionados por sus novedosas instituciones ambientales, han pasado a tomar un lugar ubicuo en los procesos productivos dentro de las comunidades nativas Yánesha. Su mediación en los procesos de inserción al mercado de la población Yánesha y el propio curso que esta le otorgue puede levantar preguntas acerca de los cambios en la gobernanza, el uso del suelo y de los recursos en el Palcazú, la Selva Central o la Amazonía peruana; pero sobre todo, permite interrogarse acerca del modo de relacionamiento contemporáneo de los pueblos indígenas amazónicos con los ríos, las pampas, los bosques y sus habitantes no-humanos ante el aluvión de otros seres -colonos, ONG, instituciones estatales, entre otros- que continuamente se transforman en este campo de la vida social. Posiblemente, la interrogante más significativa -y amplia- tras el análisis histórico y exploratorio presentado recaiga sobre el contenido de los nexos entre los últimos cambios 'económicos' (continuos pero vertiginosos) que llevan a cabo las poblaciones indígenas de la Amazonía y los sistemas de disposiciones socioculturales con los cuales rigen su vida en la floresta. 


\section{Bibliografía}

Arisi, B. M. (2012). Amazonian Exchanges: Txema's Lessons With Outboard Engines, Mosquito Nets and Images. International Review of Social Research, 2(1), 173-190.

Barclay, F. (1991). Amazonía, 1940-1990 : el extravío de una ilusión. Roma; Lima: Terra Nuova ; PUCP; CISEPA. Benavides, M., \& Pariona, M. (2002). La cooperativa forestal Yanesha y el sistema de manejo forestal comunitario en la selva central peruana. In R. C. Smith \& D. Pinedo (Eds.), El cuidado de los bienes comunes: gobierno y manejo de los lagos y bosques en la Amazonía (pp. 305-333). Lima: Instituto de Estudios Peruanos, Instituto del Bien Común.

Bourdieu, P. (2008). El sentido práctico. Madrid: Siglo Veintiuno.

Brightman, M. (2012). Maps and clocks in Amazonia: the things of conversion and conservation. The Journal of the Royal Anthropological Institute, 18(3), 554-571.

Calderón Pacheco, L. (2012). Imagenes de Otredad y de Frontera: Antropología y Pueblos Amazónicos. In C. I. Degregori (Ed.), No Hay País Más Diverso. Compendio de Antropología Peruana. (Segunda ed, pp. 235-277). Lima: Instituto de Estudios Peruanos.

Chaumeil, J.-P. (2010). Historia de Lince, de Inca y de Blanco. La percepción del cambio social en las tradiciones amerindias. Maguaré, 24, 59-67.

Descola, P. (1988). La selva culta : simbolismo y praxis en la ecología de los Achuar. Quito: Ediciones Abya-Yala; Instituto Francés de Estudios Andinos.

García-Calderón, L., Huerto, G., Quispe, E., \& Tamariz, K. (2011). El mercado y los retos de la economía yanesha. In E. Toche Medrano (Ed.), Perú hoy: ajustes al modelo económico: la promesa de la inclusión (pp. 195-211). Lima: desco.

Godelier, M. (1990). Lo ideal y lo material : pensamiento, economías, sociedades. Madrid: Taurus.

Gow, P. (2007). La ropa como aculturación en la Amazonía peruana. Amazonía Peruana, 15(30), 283-304.

Hamlin, C. C., \& Salick, J. (2003). YANESHA AGRICULTURE IN THE UPPER PERUVIAN AMAZON: PERSISTENCE AND CHANGE FIFTEEN YEARS DOWN THE 'ROAD.' Economic Botany, 57(2), 163.

Hartshorn, G., \& Stocks, A. (1993). The Palcazu project: forest management and native Yanesha communities. Journal of Sustainable Forestry, 1(1), 111-135. 
Hirsch, E. (2005). Environment and economy: mutual connections and diverse perspectives. In J. G. Carrier (Ed.), A Handbook of Economic Anthropology (pp. 370-386). Edward Elgar Publishing.

Larsen, P. B. (2016). Derechos indígenas, gobernanza ambiental y recursos en la Amazonía peruana : hacia una antropología de la posfrontera. Lima: Instituto de Estudios Peruanos.

Luján, S. (2017). Una aproximación al cambio en el uso de los recursos y las actividades económicas: la pesquería y la ganadería en la Comunidad Nativa "Loma Linda" del distrito de Palcazu-Pasco. Pontificia Universidad Católica del Perú.

Ortner, S. B. (1984). Theory in Anthropology since the Sixties. Comparative Studies in Society and History, 26(1), 126-166.

Salick, J. (1989). Bases ecológicas de los sistemas agrícolas amuesha. Amazonia Indigena, 9(15), 3-16.

Santos Granero, F. (1992). The dry and the wet: astronomy, agriculture and ceremonial life in western amazonia. Journal de La Société Des Américanistes, 78(2), 107-132.

Santos Granero, F. (1994). El poder del amor: Poder, conocimiento y moralidad entre los amuesha de la Selva Central del Perú. Lima: Centro Amazónico de Antropología y Aplicación Práctica.

Santos Granero, F. (2004). Los Yánesha. In F. Santos Granero \& F. Barclay (Eds.), Guía Etnográfica de la Alta Amazonía. Volumen IV (pp. 159-359). Instituto Francés de Estudios Andinos.

Santos Granero, F., \& Barclay, F. (1995). Órdenes y desórdenes en la Selva Central: Historia y economía de un espacio regional. Lima: Instituto Francés de Estudios Andinos.

SERNANP (Ed.). (2012). Reserva Comunal Yanesha : plan maestro 2011-2016. Lima: SERNANP.

Smith, R. C. (1983). Las comunidades nativas y el mito del gran vacío amazónico : un análisis de planificación para el desarrollo en el proyecto especial Pichis Palcazu. Lima: AIDESEP.

Smith, R. C., \& Wray, N. (1996). Amazonía: Economía indígena y mercado, los desafíos del desarrollo autónomo. Quito: Coordinadora de las Organizaciones Indígenas de la Cuenca Amazónica, Oxfam América.

Staver, C., Simeone, R., \& Stocks, A. (1994). Land Resource Management and Forest Conservation in Central Amazonian Peru: Regional, Community, and FarmLevel Approaches among Native Peoples. Mountain Research and Development, 14(2), 147-157. 
Valadeau, C., Castillo, J. A., Sauvain, M., Lores, A. F., \& Bourdy, G. (2010). The rainbow hurts my skin: Medicinal concepts and plants uses among the Yanesha (Amuesha), an Amazonian Peruvian ethnic group. Journal of Ethnopharmacology, 127(1), 175-192.

Viveiros De Castro, E. (2004). Perspectivismo y multinaturalismo en la América indígena. In A. Surrallés \& P. García Hierro (Eds.), Tierra adentro : territorio indígena y percepción del entorno (pp. 37-80). Copenhague: IWGIA.

Walker, H. (2012). Demonic trade: debt, materiality, and agency in Amazonia. Journal of the Royal Anthropological Institute, 18(1), 140-159.

Wilk, R. R., \& Cliggett, L. C. (2007). Economies and cultures : foundations of economic anthropology. Nueva York: Westview Press.

Zanotti, L. (2016). Radical Territories in the Brazilian Amazon : The Kayapó's Fight for Just Livelihoods. Tucson: The University of Arizona Press. 